\title{
List of Numbered Chemical Structures
}

1. $\beta$-Cyclodextrin

2. Cucurbit[7]uril

3. Dibenzo-18-crown-6

4. $\quad[2.2 .2]$ Cryptand

5. Metoclopramide hydrochloride

6. Hemicarcerand

7. Oxyresveratrol

8. 1-Naphthyldiazobenzene derivative

9. Cucurbituril

10. 3-Hydroxynaphthalene-2-carboxylic acid

11. Nicarpidine

12. Tropaeolin 00

13. 5-Amino-2-mercaptobenzimidazole

14. Nicotinic acid

15. Ascorbic acid

16. Indole

17. Neutral red

18. Anthracene

19. 1,8-ANS

20. Azulene

21. 9,10-Diphenylanthracene

22. 2,6-ANS

23. Coumarin 153

24. 6-Bromo-2-naphthol

25. $\alpha$-Bromonaphthalene

26. 1-Chloronaphthalene

27. Tetramethylsilane (TMS)

28. Naproxen

29. Hexyltrimethylammonium bromide

30. Octyltrimethylammonium bromide

31. 1-Adamantyl ammonium

32. 1-Adamantane-1-carboxylate

33. Methyl viologen

34. Ferrocene derivatives

35. Ferrocenylguanadinium cation

36. Benzoic acid

37. Pentylammonium cation

38. Mianserin

39. tert-Butylcalix[n]arenes

40. Pentachlorophenol

41. Camphor

42. Atenolol

43. Baicalin

44. Propriconazole

45. Norepinephrine

46. 18-Crown-6 ether 
47. $\mathrm{Cu}(\mathrm{II})$ cyclam

48. $\quad N$-(3-Aminopropyl)cyclohexane

49. C-Hexyl-2-bromoresorcerinarene

50. 4-Nitrophenol

51. Curcumin

52. Alizarin Red S

53. Azo dye

54. $\alpha$-Cyclodextrin

55. $\gamma$-Cyclodextrin

56. Trimethyl- $\beta$-cyclodextrin

57. Methyl- $\beta$-cyclodextrin

58. 2,6-o-Dimethyl- $\beta$-cyclodextrin

59. 2-Hydroxypropyl- $\beta$-cyclodextrin

60. 2,3-Dimethyl- $\beta$-cyclodextrin

61. Triacetyl- $\beta$-cyclodextrin

62. Tryptophan

63. DBO

64. 7-Methoxycoumarin

65. Nile red

66. 1-Ethylnaphthalene

67. trans-Cinnamaldehyde

68. Citric acid

69. Cucurbituril

70. Glycoluril

71. 4-Methylbenzylamine

72. 2,3-Diazobicyclo[2.2.2] hept-2-ene (DBH)

73. Methyl 2-naphthalenecarboxylate (2MN)

74. Rhodamine $6 \mathrm{G}$

75. Perylene monoimide PM1

76. Dimethylglycoluril

77. Acetylcholine

78. Ethylene urea

79. Spermine

80. t-Butyl calix[4]arene

81. Toluene

82. Anisole

83. Ester-derived calix[4]arene

84. 2,3-Bis(chloromethyl)-1,4-anthraquinone

85. $p$ - ${ }^{\mathrm{t} B u}$-calix[8]arene

86. $N, N$-Dimethylindoaniline

87. Acridine red

88. Cryptophane A

89. PAMAM G4

90. 2-Naphthol

91. 1,4-Dimethoxypillar[5]arene

92. Carboxylated pillar[n]arenes

93. Sodium toluenesulfonate

94. Bambus[6]uril

95. Cyclophane CP44 
96. Bistren host

97. Methyl orange

98. $p$-( $p$-Hydroxyphenylazobenzoate),

99. c4-Methyl-2,6-dicarboxymethylphenol ( $\mathrm{CMOH})$

100. $N, N$-Dimethylaminonaphthyl-(acrylic) acid ethyl ester

101. 4-N,N-Dimethylamino cinnamaldehyde

102. Benzoic acid

103. [C4antrlm][Cl]

104. trans-4,4'-Ethylenedipyridine

105. Carbaryl

106. Carbofuran

107. Azinphos methyl

108. Lomeflaxecin

109. Diquat

110. Diazacoronand-based host

111. Diazacoronand-based sensor

112. Crown ether-based sensor

113. Cyclodextrin-based sensor

114. Adamantine carboxylic acid

115. Diaminoazobenzene

116. 2,2'-Bipyridine-3,3'-ol

117. Dihydroergotamine (DHE)

118. Rapamycin

119. Mitoxantrone

120. Phenanthriplatin 
